ILLUSTRATIONS OF THE

\section{TOLERANCE OF INJURIES OCCASIONALLY} EXHIBITED BY THE BRAIN.

\author{
By GEORGE P. MAY, M.D., Maldon.
}

THE important functions exercised by the brain in the human economy, render the various lesions incident to this organ objects of especial interest. Although of late years many valuable physiological facts in reference to the cerebro-spinal system have been brought to light, and pathological investigations have neither been few nor unimportant, it must I think be admitted that they have not rendered the symptomatology of cerebral diseases and injuries at all satisfactory, and that in no class of diseases are the diagnostic signs so variable and ambiguous. I apprehend there are few practitioners who have not occasionally felt their faith in nosological dicta, as applied to these maladies, considerably shaken, if not entirely overturned, and who have not experienced the want of satisfactory data to enable them to pronounce with any degree of certainty whether there be inflammation of the membranes or of the substance; whether ramollissement or simple effusion; whether tubercle or abscess. Dr. Abercrombie represents " the danger of being guided by system in our diagnosis of diseases of the brain, and the necessity there still is for the careful observation of facts in regard to this class of diseases." The value of his caution and recommendation must still be appreciated.

On what depends this difficulty in determining the existence and precise nature of diseases of the encephalon? The impossibility of investigating by physical examination the condition of a viscus enclosed in an unyielding bony case, and the circumstance of the brain being a symmetrical organ, whereby the general symptoms of injury are less obvious, and less to be relied on, are circumstances which to some extent may account for the difficulties which overshadow the diagnosis. But of all the obstacles to the establishment of a legitimate induction, probably the most important arelst, those cases in which are developed symptoms usually considered to indicate a morbid state of the contents of the cranium, and yet in which an examination post-mortem reveals little or no deviation from the normal structure or condition; and, 2nd, those instances of tolerance of mischief occasionally manifested by the brain, and unassociated with the existence of symptoms at all commensurate with the severity of the affection. It is difficult to assign limits to the amount of injury which the brain will sustain without life being extinguished. Fracture of the skull, rupture of the vessels, and loss of substance, occurring separately, or even conjointly in the same case, are not only compatible with existence for a time, but are now and then unaccompanied by symptoms which would lead to the expectation of so formidable an amount of injury.

The following cases which I now beg to adduce in illustration of the foregoing statement, came under my own observation, with the exception of the first, which occurred in the practice of my father:-

\section{Case I.}

N. - aged 50, a healthy man, thrown from a colt November 28th, 1833. Suspected to have been kicked by the animal on the right side of the head. He received a scalp wound in this situation, from which much blood issued. There was hæmorrhage also from the nose, mouth, and ears. The right clavicle was broken. No fracture of the skull could at this time be discovered. He remained sensible until two hours after the accident, when he became restless for a few minutes, after which total insensibility ensued, with stertorous breathing and dilated pupil. After this condition had continued twenty-four hours, a crucial incision was made through the scalp in the situation of the wound, and a fissure was observed in the parietal bone, near the squamous suture. A circle of bone was removed by the trephine, and a considerable quantity of coagulated blood was found upon the dura mater; much of this was removed at the operation, and for two days subsequently there was exudation of blood and serum from the opening. On the day after he was trephined he manifested some signs of sensibility, raised his right hand to his face, which had before been motionless, and put out his tongue when asked. On the following day he became perfectly sensible. Pulse 80 , soft. The power of voluntary motion is perfect in the right side, but the left leg and arm are paralyzed. From this time to the nineteenth day from the receipt of the injury, his progress upon the whole was favourable. He was now capable of moving the left foot, but the arm of that side remained powerless. He evinced a strong desire for more generous diet; low diet, however, was persisted in. On the nineteenth day from the date of the accident he was seized with severe pain in the left side under the sixth rib, associated with cough, much difficulty of breathing, pulse much accelerated, fever and furred tongue. There was at this time considerable purulent discharge from the ear. He was somewhat relieved by copious bleeding, blistering, \&c. He remained some days in an unsatisfactory state, the cough and dyspnoea continuing, and sank on the twenty-ninth day after the accident. The day previous to. his death pus was observed to be discharged from the mouth.

Sectio-capitis.-On dissecting back the scalp, extravasated blood was observed as in a recent injury. The dura mater was firmly attached to the calvarium, except in an area of about two inches around the part removed by the trephine; here the bone was thinned, and felt rough. There was considerable discharge of serous fluid on cutting through the dura mater. The surface of the brain was very vascular, and a small portion of coagulable Jymph was deposited beneath the arachnoid. The brain was flattened on the injured side, and the lateral sinus was much reduced in size. The choroid plexus in both ventricles highly vascular. The fracture extended from the parietal, through the petrous portion of the temporal bone, and through the alæ of 
the sphenoid bone, nearly to the parietal of the opposite side. There was no attempt at union in the fractured bones. The left lung was intimately adherent to the thoracic walls; in attempting to separate these, a large vomica in the lower lobe burst, and discharged about a pint of purulent matter.

\section{Case II.}

P. — , aged 15, thrown from a horse June 17 th, 1839. Received a lacerated scalp wound, which denuded the bone to a considerable extent, and from which more than a pint of blood flowed. There was no fracture, and his senses were unimpaired. On the fourth day he was able to come down stairs, and to exercise himself in the open air. A great portion of the wound healed by the first intention, and for three weeks the boy appeared entirely comfortable. At the commencement of the fourth week slight febrile symptoms manifested themselves, and the discharge from the wound became offensive. This condition continued without any aggravation for six days, during which time he now and then complained of headache.

February 10th.-He became suddenly comatose, and died the following day.

Sectio-capitis.-Under the scalp around the seat of injury, was an accumulation of offensive pus, a small portion of the external table of the right parietal bone was carious. On raising the calvarium two or three drachms of fotid pus escaped. The dura mater on the right side and the subjacent membranes were eroded in two places-one about the size of a shilling immediately behind the Sylvian fissure, the other more posterior and of dimensions somewhat less. The ulcers extended about three lines into the substance of the brain, their bases were hard, their edges ragged and covered with yellowish matter. A great quantity of pus and lymph was effused over the anterior lobe. The whole of this hemisphere was very vascular; corpus callosum, septum, and fornix, in an advanced stage of softening; an excavation the size of a pigeon's egg was discovered in the posterior lobe filled with fotid matter.

Every tissue in this case appeared to be the seat of some morbid action. The membranes were extensively inflamed, the cortical substance eroded to some depth, the internal structure exhibited that alteration most usually considered to be the result of inflammatory action, and abscess completed the catalogue of mischief. But until within twenty-four hours of death there was scarcely a symptom diagnostic of any of these lesions, $\mathrm{Up}$ to this period the patient retained perfect possession of his faculties, mental and physical; there was no delirium, paralysis, convulsion, or contraction of the limbs, which have been regarded by some pathologists as indicative of cerebral ramollissement.

\section{Case III.}

F., aged 29, struck in the forehead by the bursting of a gun in 1834, whereby he was knocked down and remained insensible for half an hour after the accident.
The wound healed imperfectly, and remained in an irritable condition for some time, and several small pieces of bone were discharged from it. About six months subsequent to the accident he was attacked by fits, which were reported to be of a very violent description, and from which he recovered in a few days.

On October the 4 th, 1837 , I was first requested to see him. Having gone to bed the previous night in good health, he was discovered on the floor of his room in convulsions. These were so severe, that it required the efforts of several men to hold him. The fits occurred at intervals of about a quarter of an hour, and lasted from three to five minutes at a time. Their continuance was marked by the most violent contraction of every visible muscle; the face swelled and livid; and much foam ejected from the mouth. In the intervals he lay in a semi-comatose condition, with breathing somewhat stertorous. After copious depletion the convulsions became less violent, and in twenty-four hours had ceased entirely. He soon recovered, resumed his employment, as an agricultural labourer, and remained without any particular ailment for nearly four succeeding years. On March the 4 th, 1841, my attendance was again requested, as he had experienced a return of his old disorder. The symptoms were similar to those already detailed, but more distortion of the muscles of the face was observed. The convulsions continued at intervals for two lays, when he became comatose, and died sixty hours after the seizure.

Sectio-capitis.-A slight scar indicating the seat of the injury received by the bursting of the gun, was visible a little above the right superciliary ridge. Corresponding with this, and immediately above the frontal sinus, a piece of the frontal bone nearly the size of a sixpence was found fractured. It had suffered little displacement; its edges were smooth, as were those of the surrounding bone. No attempt at union could be perceived. A spicula, half an inch in length, evidently a portion of the internal table, protruded from the fractured piece horizontally into the brain, or rather into an excavation in the organ the size of a blackbird's egg. The dura mater terminated abruptly at the edge of the cavity. Three or four spiculæ of smaller size and of new formation, were observed shooting from the inner table around the fractured part. There was a copious effusion of lymph under the arachnoid, and bloody serum in the ventricles.

In this instance there can be no doubt that the dura mater and brain were lacerated so long back as the date of the accident-a period of seven years-and by the protruding spicula a constant source of irritation must have been kept up, which ended ultimately in the total destruction of that portion of the organ in its immediate locality.

\section{Case IV.}

A man of stout build, having the appearance of a " navvie," called at my house, August 9th, 1848. His gait was observed to be staggering : this, with some incoherence in his expression, and the appearance of a severe black eye, created a suspicion in the mind of 
Mr. Millington, my assistant, that he had been engaged in a drunken quarrel. He complained of much pain in the head, and said he had been ill-treated two days before by some men a few miles from the town. In the evening he was feverish and restless. On the following morning he was better, ancl appeared relieved by the copious action of a cathartic which was given him. He came down stairs and smoked a pipe and drank some porter in company with the frequenters of the inn where he was lodging. He was again restless at night and was found insensible in the morning, with convulsive twitchings of the muscles of the face, the limbs were entirely undisturbed. He was bled largely, in connection with the employment of other means, but he speedily sunk into a state of coma, and died early on the following morning.

Sectio-capitis, August 13.-Considerable extravasation around the right orbit. No other contusion or wound discovered. The superior surface of the brain exhibited a highly congested appearance. The inferior surface of the right anterior lobe bore marks of a recent laceration, about an inch in length; the substance of the organ around was dark and pulpy. There was an exudation of blood in this situation, diffused in a semi-coagulated state beneath the anterior and middle lobe over the orbitar plate of the frontal bone, and the sphenoid and temporal bones of that side. A fracture was observed transversely across the petrous portion of the temporal bone, extending through the occipital to the length of about four inches in the whole. In the immediate vicinity of the fracture, between the dura mater and the occipital bone of the same side, a copious extravasation of blood had taken place from rupture of the lateral sinus, the course of which was crossed by the fracture. About an ounce of strawcoloured fluid was found at the base of the brain, and a similar quantity in each of the lateral ventricles. The floors of the ventricles, and the thalami optici appeared to be preternaturally softened. A deposit of lymph was observed on the pons varolii, and on the medulla oblongata.

This case became the subject of judicial inquiry. It appeared probable, that the deceased being intoxicated, attacked with a sickle a fellow labourer who, in selfdefence, struck him a violent blow on the head with his fist and knocked him down. He was found three hours after lying insensible in the place where he fell. He was left there, but soon after got up and walked of his own accord to Waldon, a distance of between two or three miles. $\mathrm{He}$ was incapable of giving any distinct account of himself, or of the injury he had received. He came to the Castle Inn on the 7 th, complained of headache, but made no application for medical assista nce until the 9 th, as above detailed, and then only at the urgent desire of the landlady. The fracture of the skull, laceration of the brain, and rupture of the lateral sinus. occurred doubtless at one and the same time, as the result of the blow, or the consequent fall. In spite of these lesions he was able to walk some distance, to smoke and to drink, and for the first three or four days after the reception of the injuries there were no symp- toms to lead to a suspicion of the mischief which was revealed by the examination post-mortem.

In this case the effusion of blood from the lacerated vessels was in all probability slight in the first instance, but gradually increased until the amount of pressure became sufficient to destroy the functions of the brain.

December 1, 1849.

\section{CASE OF MALFORMATION,}

(Read at the Anniversary Meeting of the Association, held at Worcester, August 1st and ind, 1849.)

By JOHN JONES, Esq., M.R.C.S., Derby.

April, 1848.-Mrs. B. was delivered at the full period of pregnancy, of a remarkably large and apparently fine child. On examination, however, the following malformation was discovered. The space between the epigastrium and umbilicus is unusually long, and that between the umbilicus and pubes correspondingly short. Below the umbilicus there is a deficiency of the abdominal muscles and integuments, extending to the pubes, and leaving an opening, which at first seemed to be occupied by granulations, but was found principally to be filled up by an oval-shaped body, soft and receding on pressure, and when pressed upon a fluid escaped, proving it to be the urinary bladder, the exposed surface of which was exterior to the peritoneal reflexion, was vascular, and could with difficulty be distinguished from the surrounding granulations. Below the pubes is a portion of a penis, consisting only of the inferior half of that organ, as if divided by a longitudinal section, leaving exposed the floor of the urethra situated between the two halves of the corpora cavernosa, which terminate in two tubercular-shaped discs formed by the inferior half of the glans penis. The orifice of the urethra is a small opening situated under the symphisis pubis, and can only be discovered by pressing on the bladder, when urine is observed to escape from it. Lacunæ are also visible on the exposed surface of the urethra. The scrotum containing the testis appears to be natural; but the anus is situated unusually near to the scrotum.

The following is the present appearance of the malformation:-The preternatural opening is much contracted; the bladder is less vascular, occupies a much smaller space than at first, and seems more intimately united with the surrounding parts. The urine, of course, continues to ooze from the urethral orifice, but seems to cause little or no irritation, and with care, the little fellow is kept tolerably comfortable. I think some mechanical contrivance might be devised which would obviate in some measure the inconvenience arising from the involuntary flow of urine.

The following circumstances occurred during pregnancy, which, in the mother's estimation, sufficiently account for the malformation:-About the fourth month of gestation she was greatly alarmed in consequence of a hay-stack belonging to her husband having been burnt. At the same time her daughter, about seven years old, was affected with chronic glandu- 